\title{
Laparoscopic Surgery in Genitourinary Cancer Treatment
}

\author{
March Villalba José Antonio \\ Additional information is available at the end of the chapter \\ http://dx.doi.org/10.5772/55448
}

\section{Introduction}

Genitourinary cancer comprises kidney, prostate, bladder, upper urinary tract and testis neoplasms. The incidence rates of them vary between 15 and $1.5 \%$ in developed countries.

The epidemiology of genitourinary cancer varies depend on the organ. Prostate cancer is the most common solid neoplasm in males (15\%) and renal cell neoplasm involves since 3\% of all adult cancers. Urothelial carcinomas are the fourth most common tumors, after prostate, breast (females), lung and colorectal cancer. In particular, bladder cancer is the 9th most common cancer diagnosis worldwide. Testicular cancer is the less common genitourinary cancer that represents between $1 \%$ and $1.5 \%$ of male neoplasms [1].

The wide range of treatments against these diseases comprises surgery, radiotherapy and chemotherapy. Radiotherapy is used to treat localized and locally-advanced prostate cancer even with curative intent. Also radiotherapy is used to prophylactic treatment in seminomatous testis cancer to avoid para-aortic or iliac lymphatic relapses. In bladder cancer, radiotherapy is used as a palliative treatment against hematuria. Radiotherapy does not play an important role in kidney cancer, only it is used to treat selected metastasis cases.

Chemoteraphy in prostate cancer (Taxanes) is reserved for the treatment of metastatic castration refractory prostate cancer. At renal cell carcinoma, Tyrosine kinase inhibitors or Mammalian target of rapamycin (mTOR) inhibitors should be considered as first- or secondline treatment for metastatic disease. Cisplatin-based chemoteraphy in bladder cancer is considered as a neoadyuvant or adyuvant treatment before and after cystectomy if there is suspicion or evidence of lymph node metastasis. Carboplatin-based chemoteraphy is used to treat several seminomatous testis neoplasms stages after orchiectomy. Cisplatin, eposide and bleomycin or eposide and cisplatin combinations are used to treat non-seminomatous testis 
neoplasm combined or not to retroperitoneal lymph node nerve-sparring surgery and also in metastatic cases.

More and more genitourinary cancers are diagnosed in localized stages, making surgical treatment possible [1]. Since Bill Schuessler performed the first laparoscopic lymphadenectomy in a patient with localized prostate cancer (October 1989), Urologists have acquired technology advances applied to laparoscopic surgery. In the last decades, those advances have made a minimally invasive approach to treat these cancers easier. That has caused that today laparoscopy approach is the technique of choice in the surgical treatment of some localized genitourinary cancers [2].

According the PUBMED database, there is an increase in the publication of articles dealing with the laparoscopic treatment. In the last 5 years almost $10 \%$ of those articles referring specifically to laparoscopic surgery. (Graphic 1).

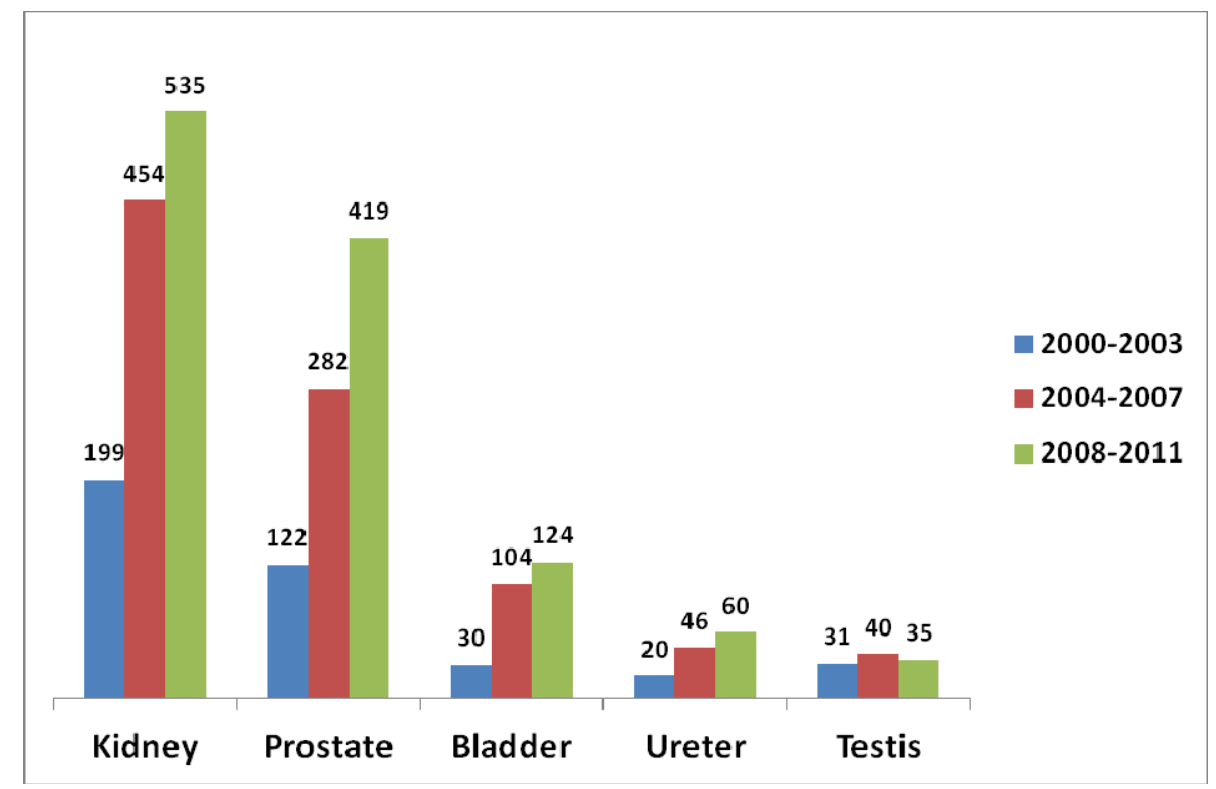

Figure 1. Evolution of published articles on laparoscopic surgery in PUBMED (MESH database).

Today there is a trend towards minimally invasive surgery but it is necessary to establish whether the outcomes of competing treatment options (open Vs laparoscopy) are comparable, focusing in postoperative morbidity and oncologic outcomes.

This chapter gives a summary of laparoscopic treatment reviewing the indication, morbidity and oncologic and functional results compared to conventional surgery for each of the listed cancers, providing a general overview. 


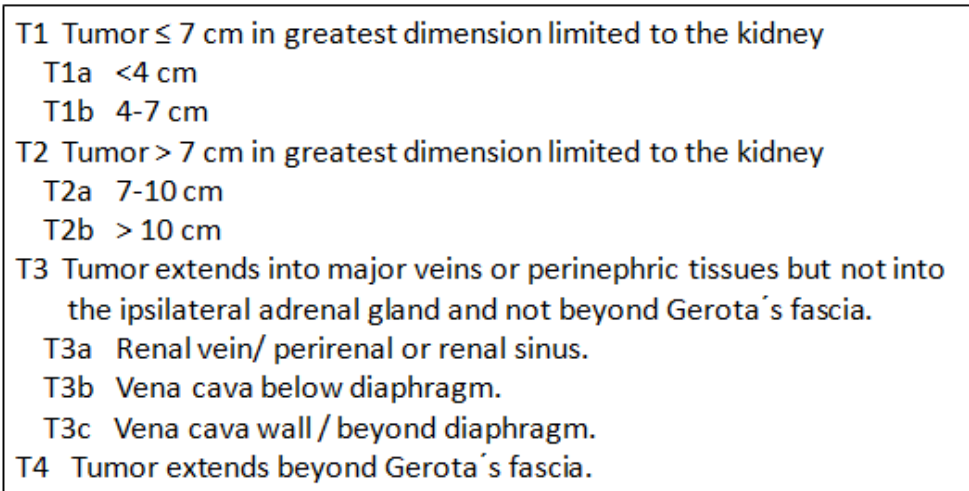

Table 1. Kidney. Primary tumor stage (T) [41].

\section{Renal cancer}

Renal cell carcinoma (RCC) accounts for $2-3 \%$ of all adult cancers. More than $50 \%$ are diagnosed at a localized stage (pT1-pT2) (Table 2). Open radical or partial nephrectomy has been the standard curative intervention for localized RCC for the past five decades, laparoscopy also being an alternative in RCC with renal vein tumor thrombus (pT3a) (Table 2). With the new minimally invasive approaches, laparoscopic radical or partial nephrectomy has become an acceptable alternative to open surgery [3,4]

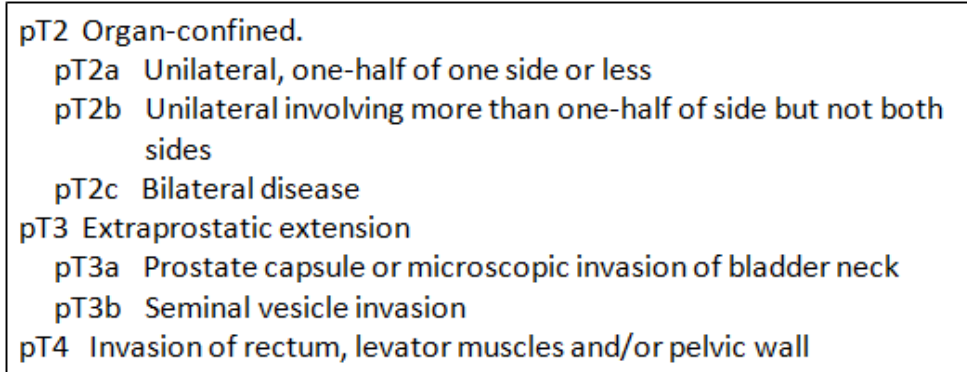

Table 2. Prostate. Pathologic tumor stage (pT) [42].

\subsection{Morbidity and functional outcomes}

In comparison with open radical nephrectomy, laparoscopic procedure offers less morbidity (back pain and postoperative blood loss) and hospital stay [3]. 
Nephron-sparing surgery offers better preservation of renal function than radical nephrectomy and lower risk of cardiac death but efforts should be made to limit the renal function loss associated with surgery for localized renal masses regarding transient ischemia at surgery, because warm ischemia time seems to be the most important independent variable for predicting renal damage. This damage occurs within the third postoperative month $[5,6]$.

Laparoscopic nephron-sparing surgery provides less blood loss, median operative time, median analgesic requirement, hospital stay and median convalescence time, compared to open partial nephrectomy. But it means more major intra-operative complications ( $5 \% \mathrm{Vs} 0 \%$ ), renal / urological complications (11\% Vs $2 \%)$ and warmer ischemia time [7].

\subsection{Oncologic outcomes}

\subsubsection{Localised renal cell carcinoma}

There are no randomised studies assessing oncological outcomes. Papers published found similar oncological outcomes; the 5 year overall survival for laparoscopic versus open radical nephrectomy was $87.8 \%$ and $88.7 \%$, respectively. There was no evidence of any difference in cancer-specific and recurrence-free survival at 5 year reported in the studies [4].

With respect to the approach at localized RCC, few randomized studies compared retroperitoneal with transperitoneal radical nephrectomy. Both of them were found to have a similar oncological outcomes and no incidences of positive surgical margins were reported $[4,8]$.

When laparoscopic partial nephrectomy was compared to open partial nephrectomy, a database review of Lane et al. noted an overall survival benefit increase in laparoscopic versus open partial nephrectomy when adjusting for age, gender, race, Charlson index, tumor size, hypertension and the predicted risk of recurrence at 5 year in those patients with a minimum of 1 year follow-up, but there were no differences in 3 year cancer-specific survival, 5 year overall survival and 7 year follow-up $(92.7 \%$ Vs $95.6 \%$ in cancer-specific survival respectively). This study described a lower risk of all-cause death in the laparoscopic group [9]. The same results were described by Gill et al. and Marszalek et al; both of them did not find differences in the recurrence patterns between both groups [10,11].

There has been controversy about the suitable tumor size to perform a laparoscopy nephronsparing intervention. A cut-off of $4 \mathrm{~cm}$ has been recommended but some authors have argued that partial nephrectomy is feasible up to $7 \mathrm{~cm}$ with no reduction in oncologic outcomes. In this view Simmons et al. published a database review for tumors larger than $4 \mathrm{~cm}$ treated by laparoscopic partial nephrectomy versus laparoscopic radical nephrectomy. There was no difference in estimated overall survival (74\% versus qué), cancer-specific survival and recurrence-free survival rates (both $81 \%$ versus qué) [4,12].

In addition to size, other factors about renal mass anatomy such as growth pattern (endo-/ meso-/exophytic) and location (central/hilar/peripheral, anterior/posterior, lateral/medial, polar) are important to consider a nephron-sparing surgery. It is more feasible if tumor is placed in a peripheral/polar/posterior site, for example [13]. 


\subsubsection{Locally-advanced renal cell carcinoma}

Laparoscopic surgery has been extended to patients with renal cell carcinoma associated with limited local invasion and lymph node metastases or in the presence of renal vein and inferior vena cava thrombi. In well-selected patients with metastatic renal cell carcinoma, laparoscopic cytoreductive nephrectomy can be performed safely, with less morbidity than open nephrectomy [14]. Laparoscopic nephrectomy in metastatic RCC should be recommended to those patients with a good performance status before oncological treatment. The expanding indications for laparoscopic radical nephrectomy are: larger tumors $(>7 \mathrm{~cm})$, renal vein tumor thrombus, cytoreductive nephrectomy and limited locally invasive tumors into psoas or diaphragm muscle. This technique must be performed in selected patients $[8,15]$.

\subsection{Conclusions}

For localized renal cancer, laparoscopic radical nephrectomy is the approach of choice because it offers less morbidity than open nephrectomy and both of them achieve similar oncological outcomes, such as survival and recurrence rates. There are no differences between transperitoneal and retroperitoneal approach.

When renal tumors are $\leq 4 \mathrm{~cm}$ laparoscopic partial nephrectomy is a good choice but location of tumor is also important to perform surgery. In these cases, partial nephrectomy improves survival.

Laparoscopic partial nephrectomy shows no improvement than laparoscopic radical nephrectomy when renal tumors are $>4 \mathrm{~cm}$ but laparoscopic approach is a correct choice depending on surgeon skill and renal mass anatomy.

For locally-advanced renal cancer, laparoscopic radical nephrectomy is a technically feasible approach in carefully selected patients with a good performance status. Optimal patient selection, large laparoscopic experience and multidisciplinary support are the more important elements for a safe application of this approach.

Additional data are needed because most of the studies are retrospective and is necessary to improve methodological quality.

\section{Prostate cancer}

Prostate cancer (PCa) is one of the most common solid neoplasm in male. In Europe it has an incidence rate of 214 cases per 1000 men, outnumbering lung and colorectal cancer. PCa is currently the second most common cause of cancer death in men [16].

Currently, there is an increase in the diagnosis of PCa, concretely clinically localized prostate cancer (table 3). Radical prostatectomy is a common treatment for these patients, who have also life expectancy more than 10 years [17]. Radical prostatectomy has been associated with complications and sequel, including intraoperative blood loss, postoperative urinary incontinence and erectile dysfunction. With the intent of reducing the invasiveness of traditional open 
retropubic approach and complications, urologists have developed the laparoscopic technique, which represents a different perspective of surgical anatomy that implies an important learning curve [17].

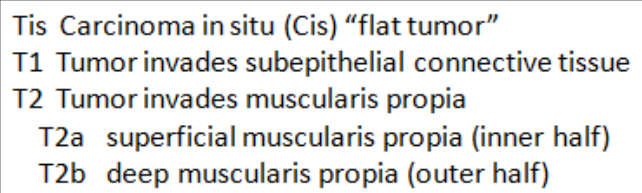

Urothelium. Histologic grade (WHO/SIUP 1993) ${ }^{43,44}$.

Grade 1 well differenciated

Grade 2 Moderately differenciated

Grade 3 poorly differenciated

Table 3. Urothelium. Primary tumor stage (T) $[43,44]$.

\subsection{Morbidity and functional outcomes}

Several studies demonstrate that operative time of laparoscopic approach was significantly longer than open retropubic approach, but laparoscopic approach showed less blood loss and lower transfusion rates than the open procedure $[17,18]$. The overall complications rate was significantly lower in those patients undergoing laparoscopy radical prostatectomy $[17,18]$.

A single, nonrandomized prospective trial compared retropubic radical prostatectomy with laparoscopy prostatectomy, demonstrating that tissue damage was significantly lower in laparoscopic approach. Specifically, plasmatic levels of IL-6 and C-reactive protein were lower at the end of procedure, 12 hours later and 24 hours later [17].

In relation to postoperative pain (measured by validated 10-point visual analogue scale), laparoscopic radical prostatectomy with retroperitoneal approach seems to be the best tolerated technique during the first five postoperative days, compared with transperitoneal and open approaches [19]. Considering the studies reporting the requirements of morphine sulfate equivalent during the postoperative course, it seems to be no differences between open and laparoscopic approach [17].

In the published comparative studies, catheterization times and hospital stay were lower in laparoscopic approach [17]. Full recovery was faster in laparoscopic than open approach [18]. Laparoscopic radical prostatectomy had a lower rate of anastomotic strictures compared to open retropubic prostatectomy [17]. 
About urinary incontinence and erectile function, cumulative analysis of the available data suggest thatcontinencerates and erectilefunction afteropen orlaparoscopicapproach are similar $[17,18]$. The same occurs when quality of life after surgery was analyzed in both groups [17].

\subsection{Oncologic outcomes}

With regard to the oncologic outcomes, in the published comparative studies, the main item evaluated was the surgical margins. Guazzoni et al published the study with the highest level of evidence, demonstrating that the positive surgical margins rates obtained after open retropubic radical prostatectomy and laparoscopic radical prostatectomy were overlapping [20]. In addition, when the data were stratified by the pathologic stages, there were no differences between the two procedures $[17,21]$.

\subsection{Conclusions}

Laparoscopy radical prostatectomy implies an important learning curve to achieve good functional and oncologic outcomes. Currently, laparoscopy approach is better than open approach in terms of perioperative and early postoperative outcomes such as: blood loss, transfusion requirements, tissue damage, postoperative pain (only the first few days), hospital stay, and full recovery time.

Several studies showed a lower rate of anastomotic stricture after laparoscopic approach.

At laparoscopic approach, the surgeon skill is very important to perform good bladder neck preservation, paraurethral dissection and nerve sparing surgery (when it is possible), to obtain good functional results. In general, laparoscopic approach does not offer more advantages in urinary continence or erectile function after surgery and the oncologic outcomes, compared to open approach, are similar.

There were no differences between retroperitoneal and transperitoneal approach [22].

It is necessary more prospective studies, as well randomised series to analyze oncologic outcomes and morbidity comparing more than retroperitoneal or transperitoneal approach at the laparoscopic procedure, as well as the addition of lymphadenectomy.

Itislikely that themostcriticalissueinsurgicaltreatmentoflocalized prostatecanceristheselection of the best surgical technique fitted to the surgeon, rather than only the surgical approach.

\section{Urothelial cancer: Bladder and upper urinary tract}

\subsection{Bladder cancer}

Bladder cancer is the 9th most common cancer diagnosis worldwide, with an estimated male: female ratio of 3.8:1.0. At the initial diagnosis of bladder cancer approximately $30 \%$ has muscleinvasive disease [23]. 
Muscle invasive urothelial bladder cancer is a highly aggressive disease in which surgical treatment is essential for survival. Although open radical cystectomy is the gold standard treatment for muscle-invasive, organ-confined bladder carcinoma, there is increasing interest in laparoscopic radical cystectomy [24,25].

Radical cystectomy is also an optional or recommended treatment in high grade tumors like T1G3 or Cis (table3) with high risk of progression and/or multiple recurrences after immunotherapy (intravesical BCG) treatment [23].

Laparoscopic radical cystectomy could be divided in three times: 1, cystoprostatectomy in males or cystectomy plus hysterectomy and ooforectomy in females; 2 , lymphadenectomy and 3, urinary device reconstruction (intra or extracorporeal).

At this surgery it is necessary to have performed a correct selection criteria, including organconfined disease $(\leq \mathrm{T} 3)$, nonbulky limphadenopathy, absence of uncorrected coagulopathy, body mass index $<35 \mathrm{~kg} / \mathrm{m}^{2}$, non severe cardiorespiratory compromise and absence of prior abdominal surgery or prior pelvic radiation therapy, because it has been shown that the patients who do not meet these criteria have higher complication rates and poor profit of lymphadenectomy [24].

About lymphadenectomy, there is strong evidence that the more nodes removed at cystectomy, the better long-term survival time, so it is very important the extent of the lympadenectomy and the number of nodes removed. At laparosopic approach, it requires high-level laparoscopic skills [24].

\subsubsection{Morbidity and functional outcomes}

General postoperative complications rate of open radical cystectomy vary between $30 \%-60 \%$ of patientsand themortalityisabout $1.5 \%$.Atlaparoscopiccystectomythereported ratesfallbetween $8 \%-42 \%$ and $1 \%$ of mortality, but these reports do not define blood transfusion as a complication [24]. Nix et al. performed the only randomized controlled trial and reported no difference in complicationsbetweenopenandlaparoscopicapproach, butthegroupsweretoosmall[26].Haber et al. in their retrospectively series ( $\mathrm{n}=50$ in each group) showed an important benefit for the laparoscopic approach and extracorporeal urinary diversion. These cases were associated with reduced bloodloss, decreased ileus, shorterhospitalstay and nodifferencesinoperating timeand post-operative complications, and appear to haveless postoperativeanalgesicrequirements [27].

The indications or nerve-sparing cystectomy are limited to selected young patients with organconfined low-volume of tumor and extratrigonal location who are keen to maintain their sexual potency. This preservation has rarely been analyzed and the series published lacked potency data. Prostate-sparing radical cystectomy, another way to preserve erection, is controversial, and there are no data about long-term oncological outcomes to validate their safety [27].

Regarding to urinary diversion, Haber et al. showed that the laparoscopic assisted urinary diversion technique provides decreases in operating time, blood loss, transfusion rate, and more rapid postoperativereturntooralintakeandambulation, althoughmajorcomplicationsrequiring re-operation occurred more in this group than in the extracorporeal reconstruction [27]. 


\subsubsection{Oncologic outcomes}

About surgical margins, both approaches obtained equivalent rates. The international Laparoscopic Cystectomy Registry has been established a surgical margins rate of $2 \%$, compared to $1.6 \%$ for patients with organ-confined disease after open radical cystectomy [24, 27]. Chade et al. published an incidence of positive surgical margins ranged from $4-5 \%$ and $0-5 \%$ in open and laparoscopic approach respectively [25].

Lymphadenectomy plays an important role in oncological outcome. Published reports showed an inferior average of nodal retrieval in laparoscopic procedure than in open approach. This procedure strongly depends on the surgeon skills [25].

Local recurrence rates also appeared similar in both groups, around 7-10\% [24]. Overall survival published in the laparoscopic series was $90-100 \%$ at $1-2$ years and $63-79 \%$ at $2-3$ years (selected cases), compared to open radical cystectomy series showing 62-68\% at 5 years [25]. It is important to consider that the majority of published series had inadequate follow-up periods or/and the laparoscopic and open cohorts were no identical (strong selection bias) [28].

Port-site recurrence seems rare with current laparoscopic techniques for placement of the specimens into a laparoscopic bag, although there are few papers published on this topic. Tanaka et al., for example, described one case of recurrence in a patient with a locally-advanced stage $(0.3 \%)$ [29].

\subsubsection{Conclusions}

Laparoscopic radical cystectomy is a difficult surgical technique specially to perform a correct pelvic lymphadenectomy. Today it cannot be considered an alternative to open approach because the postoperative series failed in selection criteria and follow-up period (not more than 5 years). Multicentre prospective trials are needed.

Hand-assisted laparoscopic radical cystectomy has a less period of learning curve but it decreases the advantages of a minimally invasive technique like pure laparoscopic cystectomy.

The extirpative component of laparoscopic cystectomy is well established. However laparoscopic lymphadenectomy and reconstruction remains challenging, time-consuming, and could be associated with major complications. Shorter number of cases demonstrated that it is preferable to perform intracorporeal construction of the urinary diversion.

In conclusion, laparoscopic radical cystectomy with urinary diversion is a difficult procedure that should be reserved for selected cases (localized bladder cancer) and performed by experienced laparoscopic surgeons in selected centers.

\subsection{Upper urinary tract urothelial cell cancer}

Urothelial carcinomas are the fourth most common tumors after prostate (males) or breast (females) cancer, lung cancer and colorectal cancer. They can be located in the upper urinary tract (pyelocaliceal cavities and ureter). Upper urinary tract carcinomas are uncommon and 
account for only $5-10 \%$ of urothelial carcinomas. In $8-13 \%$ of patients, concurrent bladder cancer is present [30].

Laparoscopic radical nephroureterectomy is reserved for localized disease (the tumor not invades beyond peripelvic or periureteric fat or renal parenchyma). This technique must comply with oncologic principles, which consists on preventing tumour seeding by avoiding drilling the upper urinary tract during resection. The excision includes the distal ureter to avoid recurrence. In the first experience there were reports of retroperitoneal metastatic or trocar dissemination when locally-advanced tumors are manipulated [30]. Laparoscopic nephroureterectomy must take a place in a closed system, tumor morcelation should be avoided and an endobag is necessary to extract the specimen (kidney and ureter removed en bloc with the bladder cuff) [30].

Open and laparoscopic access seems to be equivalent in terms of efficacy and oncologic results, but regarding to functional outcomes laparoscopy approach is superior to open surgery (as in the laparoscopy nephrectomy). Laparoscopic approach resulted in less blood loss, shorter hospital stay, decreased analgesic use, a shorter interval to oral intake, a decreased analgesic use and a decrease interval to convalescence, with no significant difference in the rate of perioperative complications $[30,31]$.

Laparoscopy lymph node dissection associated with nephroureterectomy allows for optimal staging and has a therapeutic purpose. However, the anatomic areas to perform it have not yet clearly defined and it is not possible to standardize the indications to perform an extended lymphadenectomy because the published data are retrospective [30].

Several reports analyzed retroperitoneoscopic nephroureterectomy; most of them found that this approach was associated with longer operative times. However, blood loss, analgesic use, and length of hospital stay were decreased in comparison to open approach [31].

In conclusion, laparoscopic nephroureterectomy is a feasible approach at localized upper urinary tract tumors when it is impossible to perform a conservative treatment.

\section{Testicular cancer}

Testicular cancer represents between $1 \%$ and $1.5 \%$ of male neoplasms and $5 \%$ of urological tumors in general. About $15-20 \%$ of stage I (no clinical node metastasis) seminoma and up to $30 \%$ of nonseminomatous germ cells cancer patients have subclinical metastases. Surgical resection is the gold standard for managing postchemotherapy residual retroperitoneal tumor mass cases [32,33].

Laparoscopic surgery in testicular cancer is focused on the treatment of residual retroperitoneal mass or lymph node metastasis after chemotherapy. Retroperitoneal lymph node dissection is still a diagnostic and therapeutic option mainly in stage I disease [34,33]. 


\subsection{Morbidity and functional outcomes}

Compared with open retroperitoneal lymph node dissection, laparoscopic approach, done by expert hands, showed improvements in terms of analgesic requirements, complication rate $(15.6 \% \mathrm{Vs} 33 \%)$, re-do surgery rate $(1.4 \% \mathrm{Vs} 6.6 \%)$ and hospital stay but laparoscopic approach was associated with longer operating time [34-36].

Complication rates about laparoscopic approach varied between $5.6 \%$ and $46.7 \%$. Major intraoperative complications included bleeding and ureteral, duodenal and gallbladder injury. In expert hands the vast of complications could be managed laparoscopically as low conversion rates reported (1-5.4\%). About retrograde ejaculation, a late complication, reports showed a $2-3 \%$ [34-36].

Retroperitoneal laparoscopic lymph node dissection was described by some authors. Results showed its equivalence compared to conventional transperitoneal laparoscopic approach [37].

\subsection{Oncologic outcome}

Regarding stage I nonseminomatous germ cell tumor (any stage but not lymph node invasion or metastasis), there were no differences between open and laparoscopic approach in terms of retroperitoneal relapse, distant progression, biochemical failure and in-field relapse. There were three reports of port-site metastasis $(0.3 \%)$ in the literature. The rate of positive lymph nodes was lower after laparoscopic approach. The need for secondary retroperitoneal surgery did not differ (1.1-1.5\%) and both groups showed similar cure rates (99.6\%-100\%) [34].

\subsection{Conclusion}

To justify laparoscopic approach instead of open surgery, laparoscopic retroperitoneal lymph node dissection is a safe procedure with low complication rate and with perioperative outcomes comparable with open surgery [35].

The consensus of the authors is that open or laparoscopic retroperitoneal lymph node dissection should be concentrated in dedicated referral centers. Thus laparoscopic lymph node resection might be indicated in: low-risk stages, if primary tumor contains mature teratoma or if the primary nonseminomatous germ cell tumor is marker negative. Laparoscopic retroperitoneal lymph node dissection represents a valuable tool for selected patients with clinical stage I. Further studies should focus on the curative potential of the procedures as well as on the role of post chemotherapy $[33,34]$.

Laparoendoscopic single-site and natural orifice transluminal endoscopic in Urology oncologic surgery. To minimize minimal invasive surgery.

Natural orifice transluminal endoscopic surgery (NOTES) and laparoendoscopic single-site surgery (LESS) have been developed to reduce morbidity and scarring. NOTES uses existing orifices of the human body to perform surgical or diagnostic techniques. The use of accessory transabdominal ports as a part of evolution of NOTES is defined as hybrid NOTES. LESS procedure implies only a single-port or a single-incision laparoscopy [38]. 
LESSsurgerywasperformedinseveraloncologicprocedures, sucharadicalnephrectomy, radical prostatectomy, nephroureterectomy and radical cystectomy. Cumulative series showing results have been published. LESS has shown to be feasible (in expert hands) offering patient satisfaction and shortened convalescence applied to nephrectomy and radical prostatectomy. Patient selection is the most important to minimizing complication and conversion rates [38].

Comparative series between conventional laparoscopy and LESS have been demonstrated that there were only differences about cosmetic results when LESS was applied to perform radical nephrectomy. Also, there were no differences concerning analgesia and hospital stay. So far, all the comparative series fall to offer large number of cases, and they were retrospective and norandomized [38].

About hybrid NOTES applied to oncologic surgery, the most commonly procedure performed was transvaginal radical nephrectomy. Vaginal access was only used to insert a deflectable camera, whereas two additional abdominal trocars were used as main working ports for instrumentation. Currently, there are few papers with short number of cases and it requires clinical and external validation [38].

LESS has proved to be immediately applicable in the clinical practicum, but requires a skilled laparoscopic surgeon and well-selected patients. The current benefits of LESS are limited to improve cosmetic results [39]. About NOTES, the question is whether women would prefer a transvaginal access; there were studies published that showed a negative / neutral opinion of nulliparous younger women, concerning about the effect of NOTES on sexual function $[38,40]$.

In the future, it is important to perform a standard evaluation of cosmetic results, to design prospective series combining LESS and NOTES as well as improving laparoscopic ergonomics and instrumental [40].

\section{Acknowledgements}

To Katherinne Eloise, Katherine Julia, José Jr. and my parents.

\section{Author details}

March Villalba José Antonio

European Board of Urology , Hospital Clínico Universitario de Valencia, Spain

\section{References}

[1] European Association of Urology guidelines 2012ed. http://www.uroweb.org/guidelines/online-guidelines/. 
[2] Bishoff, J. T. Kavoussi. Atlas of laparoscopic Urologic Surgery. Prologue. Bishoff JT, Kavoussi:Elsevier Masson;(2008). Barcelona.Spain.13.

[3] Maclennan, S, Imamura, M, Lapitan, M. C, Omar, M. I, Lam, T. B, Hilvano-cabungcal, A. M, et al. UCAN Systematic Review Reference Group. Systematic Review of Perioperative and Quality-of-life Outcomes Following Surgical Management of Localised Renal Cancer. Eur Urol. (2012). Jul 20. [Epub ahead of print]

[4] Maclennan, S, Imamura, M, Lapitan, M. C, Omar, M. I, Lam, T. B, Hilvano-cabungcal, A. M, et al. UCAN Systematic Review Reference Group. Systematic review of oncological outcomes following surgical management of localized renal cancer. Eur Urol. (2012). , 61, 972-993.

[5] Weight, C. J, Larson, B. T, Fergany, A. F, et al. Nephrectomy induced chronic renal insufficiency is associated with increased risk of cardiovascular death and death from any cause in patients with localized cT1b renal masses. J Urol (2010). , 183, 1317-23.

[6] Porpiglia, F, Fiori, C, Bertolo, R, Morra, I, Russo, R, Piccoli, G, et al. Long-term functional evaluation of the treated kidney in a prospective series of patients who underwent laparoscopic partial nephrectomy for small renal tumors. Eur Urol. (2012). Jul;, 62(1), 130-5.

[7] Gill, I. S, Matin, S. F, Desai, M. M, et al. Comparative analysis of laparoscopic versus open partial nephrectomy for renal tumors in 200 patients. J Urol. (2003). , 170, 64-8.

[8] Desai, M. M, Strzempkowski, B, Matin, S. F, Steinberg, A. P, Ng, C, Meraney, A. M, Kaouk, J. H, \& Gill, I. S. Prospective randomized comparison of transperitoneal versus retroperitoneal laparoscopic radical nephrectomy. J Urol. (2005). Jan;; 173(1), 38-41.

[9] Lane, B. R, \& Gill, I. S. year oncological outcomes after laparoscopic and open partial nephrectomy. J Urol. (2010). Feb;, 183(2), 473-9.

[10] Gill, I. S, Kavoussi, L. R, Lane, B. R, Blute, M. L, Babineau, D, Colombo, J. R, et al. Comparison of 1,800 laparoscopic and open partial nephrectomies for single renal tumors. J Urol. (2007). Jul;; 178(1), 41-6.

[11] Marszalek, M, Meixl, H, Polajnar, M, Rauchenwald, M, Jeschke, K, \& Madersbacher, S. Laparoscopic and open partial nephrectomy: a matched-pair comparison of 200 patients. Eur Urol. (2009). May;, 55(5), 1171-8.

[12] Simmons, M. N, Chung, B. I, \& Gill, I. S. Perioperative efficacy of laparoscopic partial nephrectomy for tumors larger than $4 \mathrm{~cm}$. Eur Urol. (2009). Jan;, 55(1), 199-207.

[13] Tsivian, M, Ulusoy, S, Abern, M, Wandel, A, Sidi, A. A, \& Tsivian, A. Renal Mass Anatomic Characteristics and Perioperative Outcomes of Laparoscopic Partial Nephrectomy: A Critical Analysis. J Endourol. (2012). Jul 30. [Epub ahead of print] 
[14] Mattar, K, \& Finelli, A. Expanding the indications for laparoscopic radical nephrectomy. Curr Opin Urol. (2007). Mar;, 17(2), 88-92.

[15] Canda, A. E, \& Kirkali, Z. Current management of renal cell carcinoma and targeted therapy. Urol J. (2006). Winter;, 3(1), 1-14.

[16] Heidenreich, A, Bastian, P. J, Bellmunt, J, Bolla, M, Joniau, S, Mason, M. D, Matveev, $\mathrm{V}$, et al. Guidelines of prostate cáncer. Avaible at: http://www.uroweb.org/gls/pdf/ 08\%20Prostate\%20Cancer_LR\%20March\%(2013). th\%202012.pdf).

[17] Ficarra, V, Novara, G, Artibani, W, Cestari, A, Galfano, A, Graefen, M, et al. Retropubic, laparoscopic, and robot-assisted radical prostatectomy: a systematic review and cumulative analysis of comparative studies..Eur Urol. (2009). May;, 55(5), 1037-63.

[18] Ghavamian, R, Knoll, A, Boczko, J, \& Melman, A. Comparison of operative and functional outcomes of laparoscopic radical prostatectomy and radical retropubic prostatectomy: single surgeon experience. Urology. (2006). Jun;, 67(6), 1241-6.

[19] Remzi, M, Klingler, H. C, Tinzl, M. V, Fong, Y. K, Lodde, M, Kiss, B, \& Marberger, M. Morbidity of laparoscopic extraperitoneal versus transperitoneal radical prostatectomy verus open retropubic radical prostatectomy. Eur Urol. (2005). Jul;, 48(1), 83-9.

[20] Guazzoni, G, Cestari, A, Naspro, R, Riva, M, Centemero, A, Zanoni, M, et al. Intraand peri-operative outcomes comparing radical retropubic and laparoscopic radical prostatectomy: results from a prospective, randomised, single-surgeon study. Eur Urol. (2006). Jul;; 50(1), 98-104.

[21] Magheli, A, Gonzalgo, M. L, Su, L. M, Guzzo, T. J, Netto, G, Humphreys, E. B, et al. Impact of surgical technique (open vs laparoscopic vs robotic-assisted) on pathological and biochemical outcomes following radical prostatectomy: an analysis using propensity score matching. BJU Int. (2011). Jun;, 107(12), 1956-62.

[22] Van Velthoven, R. F. Laparoscopic radical prostatectomy: transperitoneal versus retroperitoneal approach: is there an advantage for the patient? Curr Opin Urol. (2005). Mar; 15(2), 83-8.

[23] Stenzl, A, Witjes, J. A, Compérat, E, Cowan, N. C, De Santis, M, Kuczyk, M, Lebret, T, Ribal, M. J, et al. European urology Guidelines on Bladder Cancer Muscle-invasive and Metastatic. Avaiable in: http://www.uroweb.org/gls/pdf/07_Bladder\%20Cancer_LR\%20II.pdf

[24] Challacombe, B. J, Bochner, B. H, Dasgupta, P, Gill, I, Guru, K, Herr, H, et al. The role of laparoscopic and robotic cystectomy in the management of muscle-invasive bladder cancer with special emphasis on cancer control and complications. Eur Urol. (2011). Oct; ; 60(4), 767-75.

[25] Chade, D. C, Laudone, V. P, Bochner, B. H, \& Parra, R. O. Oncological outcomes after radical cystectomy for bladder cancer: open versus minimally invasive approaches. J Urol. (2010). Mar;, 183(3), 862-69. 
[26] Nix, J, Smith, A, Kurpad, R, Nielsen, M. E, Wallen, E. M, \& Pruthi, R. S. Prospective randomized controlled trial of robotic versus open radical cystectomy for bladder cancer: perioperative and pathologic results. Eur Urol. (2010). Feb;, 57(2), 196-201.

[27] Haber, G. P, Crouzet, S, \& Gill, I. S. Laparoscopic and robotic assisted radical cystectomy for bladder cancer: a critical analysis. Eur Urol. (2008). Jul;, 54(1), 54-62.

[28] Hautmann, R. E. The oncologic results of laparoscopic radical cystectomy are not (yet) equivalent to open cystectomy. Curr Opin Urol. (2009). Sep; 19(5), 522-6.

[29] Tanaka, K, Hara, I, Takenaka, A, Kawabata, G, \& Fujisawa, M. Incidence of local and port site recurrence of urologic cancer after laparoscopic surgery. Urology. (2008). Apr; 71(4), 728-34.

[30] Rouprêt, M, Zigeuner, R, Palou, J, Boehle, A, Kaasinen, E, Sylvester, R, et al. European guidelines for the diagnosis and management of upper urinary tract urothelial cell carcinomas: (2011). update. Eur Urol. 2011;, 59, 584-94.

[31] Ristau, B. T, Tomaszewski, J. J, \& Ost, M. C. Upper tract urothelial carcinoma. Current treatment and outcomes. Urology (2012). , 4, 749-56.

[32] Albers, P, Albrecht, W, Algaba, F, Bokemeyer, C, Cohn-cedermark, G, Fizazi, K, et al. Testicular cancer guidelines. European guidelines (2011). Avaiable at: http:// www.uroweb.org/gls/pdf/10_Testicular_Cancer.pdf.

[33] Öztürk, Ç, Van Ginkel, R. J, Krol, R. M, Gietema, J. A, Hofker, H. S, \& Hoekstra, H. J. Laparoscopic resection of a residual retroperitoneal tumor mass of nonseminomatos testicular germ cell tumors. Surg Endosc (2012). , 26, 458-67.

[34] Rassweiler, J. J, Scheitlin, W, Heidenreich, A, Laguna, M. P, \& Janetschek, G. Laparoscopic retroperitoneal lymph node dissection: does it still have a role in the management of clinical stage I nonseminomatous testis cancer? A European perspective.

[35] Hyams, E. S, Pierorazio, P, Proteek, O, Sroka, M, Kavoussi, L. R, \& Allaf, M. E. Laparoscopic retroperitoneal lymph node dissection for clinical stage I nonseminomatous germ cell tumor: a large single institution experience. J Urol (2012). , 487-92.

[36] Steiner, H, Zangerl, F, Stöhr, B, Granig, T, Ho, H, Bartsch, G, et al. Results of bilateral nerve sparing laparoscopic retroperitoneal lymph node dissection for testicular cancer. J Urol (2008). , 1348-53.

[37] Arai, Y, Kaiho, Y, Yamada, S, Saito, H, Mitsuzuka, K, Yamashita, S, et al. Extraperitoneal laparoscopic retroperitoneal lymph node dissection after chemotherapy for nonsemonimatous testicular germ-cell tumor:surgical and oncological outcomes. Int Urol Nephrol (2012).

[38] Autorino, R, Cadeddu, J. A, Desai, M. M, Gettman, M, Gill, I. S, Kavoussi, L. R, et al. Laparoendoscopic single-site and natural orifice transluminal endoscopic surgery in urology: a critical analysis of the literature. Eur Urol (2011). , 59, 26-45. 
[39] Oh, T. H. Current status of laparoscopic single-site surgery in urologic surgery. Kor J Urol (2012). , 53, 443-50.

[40] Rassweiler, J. J. Is LESS/NOTES relay more? Eur Urol (2011). , 59, 46-50.

[41] Edge, S. B, Byrd, D. R, Compton, C. C, Fritz, A. G, Greene, F. I, \& Trotti, A. Kidney.Cancer staging handbook AJCC. Seventh ed. Chicago IL: Springer; (2010). , 73-86.

[42] Edge, S. B, Byrd, D. R, Compton, C. C, Fritz, A. G, \& Greene, F. I. Trotti A Prostate. Cancer staging handbook AJCC. Seventh ed. Chicago IL: Springer; (2010). , 51-64.

[43] Edge, S. B, Byrd, D. R, Compton, C. C, Fritz, A. G, Greene, F. I, \& Trotti, A. Urinary Bladder Cancer staging handbook AJCC. Seventh ed. Chicago IL: Springer; (2010). , 95-103.

[44] Edge, S. B, Byrd, D. R, Compton, C. C, Fritz, A. G, Greene, F. I, \& Trotti, A. Renal pelvis and Ureter Cancer staging handbook AJCC. Seventh ed. Chicago IL: Springer; (2010). , 87-93. 